\title{
Effects of Light Quality on The Quality Formation of Tomato Fruits
}

\author{
Baoxing XIE \\ College of Horticulture \\ South China Agricultural University \\ Guangzhou 510642, China \\ e-mail: liuhch@scau.edu.cn \\ Houcheng LIU* \\ College of Horticulture \\ South China Agricultural University \\ Guangzhou 510642, China \\ e-mail: liuhch@scau.edu.cn
}

\author{
Shiwei SONG \\ College of Horticulture \\ South China Agricultural University \\ Guangzhou 510642, China \\ e-mail: liuhch@scau.edu.cn
}

\author{
Guangwen SUN \\ College of Horticulture \\ South China Agricultural University \\ Guangzhou 510642, China \\ e-mail: liuhch@scau.edu.cn
}

\author{
Riyuan CHEN \\ College of Horticulture, \\ South China Agricultural University \\ Guangzhou 510642, China \\ e-mail: liuhch@scau.edu.cn
}

\begin{abstract}
The effects of light quality on the quality formation of tomato (cv. MicroTom) fruits were studied in controlled-environment house. There were 4 LEDs treatments: the ratio of red $(660 \mathrm{~nm})$ : blue $(460 \mathrm{~nm})$ was $1: 1(1 \mathrm{R} 1 \mathrm{~B}), 2: 1$ (2R1B), 4:1 (4R1B) and 8:1 (1R1B), the Photosynthetic Photon Flux Density (PPFD) was $300 \mu \mathrm{mol} \cdot \mathrm{m}^{-1} \mathrm{~s}^{-1}$ and illumination time was 6:00 - 18:00, the quality of tomato fruits were determined at 30, 45 and 60 days after anthesis (DAA). The contents of soluble protein, free amino acid, flavonoid and ferric reducing antioxidant potential (FRAP) in tomato fruits significantly increased under the 1R1B treatment with the development of tomato fruits. Under 4R1B treatment, the contents of soluble sugar significantly increased at 30 and $40 \mathrm{DAA}$, the rate of DPPH free radical scavenging significantly increased at 30 DAA. The polyphenol contents significantly increased at 45 and 60 DAA under 4R1B or 8R1B treatment. The lycopene contents significantly increased at 45 and 60 DAA under 2R1B treatment.
\end{abstract}

Keywords-red light; blue light; tomato; quality formation

\section{INTRODUCTION}

Light (light intensity, illumination time and light quality) is the most important factor for plant growth and development. Light is energy source for photosynthesis and an important environmental signal for plant growth and development. The combination of red and blue LED light is one of efficient light source for plant growth and development as well as improving the quality of plant nutrition $[1,2]$.

The contents of soluble sugar and vitamin $\mathrm{C}$ in lettuce increased under red light, and increased with the ratio of red light increased [3]. And soluble sugar content of lettuce significantly increased under the combination of red and blue light compared with monochromatic light [4, 5]. The contents of free amino acids and soluble protein in alfalfa sprouts [6] and Chinese toon sprouts [7] increased in blue light treatment. There were higher contents of phenol and flavonoids in lettuce under the combination of higher blue light, compared with the red light and control [8, 9]. There was the highest lycopene content in tomato fruits at 60 days after anthesis under red light LED treatment [10].

Tomatoes (Solanum lycopersicum L.) are one of the most widely consumed vegetables around world. Synergistic interactions between various phytonutrients (carotenoids, lycopene, total phenolics, etc.) in tomatoes are responsible for its beneficial health effects. Tomato has been conferred as a "functional and nutraceutical food" [11]. The functional quality and antioxidant constituents of tomato are significantly affected by environmental factors and genetics. The nutritional quality change during tomato fruit development under different ratio of red and blue LED light was determined in this study.

\section{MATERIAL AND METHODS}

\section{A. Plant Material Cultivation}

The experiment was carried out in a controlled environment house in South China Agricultural University, $24-26^{\circ} \mathrm{C} / 15-18^{\circ} \mathrm{C}$ (day/night), relative humidity (RH) $60 \%-80 \%$. The development of tomato fruit could be divided into four stages: immature green (15 days after anthesis, DAA), light-pink (30 DAA), red mature (45 DAA), and full mature (60 DAA). The tomato seedlings with 4 leaves 
transplanted in hydroponic, nutrient solution including $\mathrm{N} 200$ $\mathrm{mg} / \mathrm{L}$, P $30 \mathrm{mg} / \mathrm{L}, \mathrm{K} 240 \mathrm{mg} / \mathrm{L}, \mathrm{Ca} 130 \mathrm{mg} / \mathrm{L}, \mathrm{Mg} 48 \mathrm{mg} / \mathrm{L}$, Fe $2 \mathrm{mg} / \mathrm{L}$, B $0.5 \mathrm{mg} / \mathrm{L}, \mathrm{Mn} 0.5 \mathrm{mg} / \mathrm{L}, \mathrm{Zn} 0.05 \mathrm{mg} / \mathrm{L}, \mathrm{Cu} 0.02$ $\mathrm{mg} / \mathrm{L}$, Mo $0.02 \mathrm{mg} / \mathrm{L}$. The nutrient solution was refreshed every 9 days. There were 4 LEDs treatments: the ratio of red (660nm): blue $(460 \mathrm{~nm})$ was 1:1 (1R1B), 2:1 (2R1B), 4:1 (4R1B) and 8:1 (81R1B). The Photosynthetic Photon Flux Density (PPFD) was $300 \mu \mathrm{mol} \cdot \mathrm{m}^{-1} \mathrm{~s}^{-1}$ and illumination time was 6:00 - 18:00.

The tomato fruits were sampled at 30,45 and 60 DAA and stored under $-4^{\circ} \mathrm{C}$ for analysis.

\section{B. $\quad$ Fruit Quality Determined}

The contents of soluble sugar were measured with anthrone colorimetric method according to [12]. The contents of soluble protein were measured with Coomassie brilliant blue method according to [12]. The contents of free amino acid were determined with ninhydrin coloration method according to [12]. The lycopene contents were measured by spectrophotometer according to [13]. The polyphenol contents were determined by the Folin-Ciocalteu procedure according to [14]. The flavonoid content was measured referring to [15]. The DPPH radical-scavenging activity was estimated following the method of [14]; FRAP was determined according to the method described by Benzie et al. (1999) [16].

Data were analyzed by SPSS System (SPSS 10.0, SPSS Inc). Mean separations were conducted using a LSD test protected by ANOVA (Analysis of Variance) at $\mathrm{p}<0.05(\mathrm{n}=$ $5)$.

\section{RESULTS}

\section{A. Effect of Light Quality on The Contents of Soluble Sugar in Tomato Fruit}

With the tomato fruits development, the contents of soluble sugar continuously and significantly decreased, especially in 4R1B treatment (Fig. 1). The contents of soluble sugar significantly increased at 30 and 45 DAA under 4R1B treatment, while increased at 60 DAA under 8R1B treatment. At $30 \mathrm{DAA}$, the contents of soluble sugar in 4R1B treatment were $13.02 \%, 25.20 \%$ and $36.84 \%$ higher than $8 \mathrm{R} 1 \mathrm{~B}, 2 \mathrm{R} 1 \mathrm{~B}$ and 1R1B treatment respectively. And at 45 DAA, the contents of soluble sugar in 4R1B treatment increased $12.90 \%$ and $19.26 \%$ than $2 \mathrm{R} 1 \mathrm{~B}$ and $1 \mathrm{R} 1 \mathrm{~B}$. While at $60 \mathrm{DAA}$, the contents of soluble sugar in 8R1B treatment increased by $11.10 \%, 14.86 \%$ and $22.09 \%$ than $1 \mathrm{R} 1 \mathrm{~B}, 4 \mathrm{R} 1 \mathrm{~B}$ and $2 \mathrm{R} 1 \mathrm{~B}$ respectively.

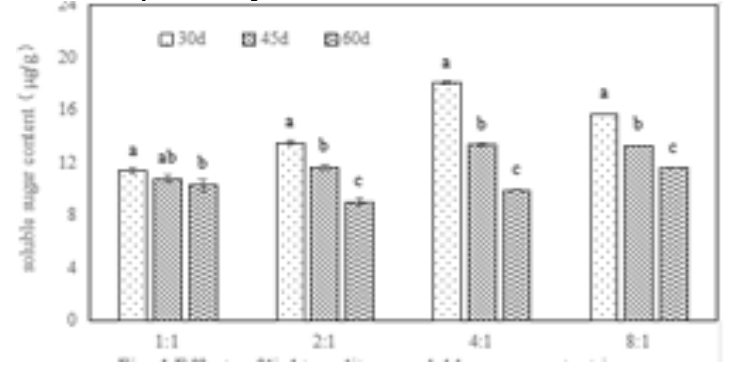

Figure 1. Effects of light quality on soluble sugar content in tomato fruit

\section{B. Effect of Light Quality on The Content of Soluble Protein in Tomato Fruit}

With the development of tomato fruits, the contents of soluble protein increased under 4R1B treatment (Fig. 2), while those in other treatments increased and then decreased. During the development of tomato fruits, the contents of soluble protein significantly increased at 45 and 60 DAA under 1R1B treatment, while significantly increased at 30 DAA under 8R1B treatment. At 30 DAA, the content of soluble protein in $8 \mathrm{R} 1 \mathrm{~B}$ treatment increased by $8.17 \%$, $17.30 \%$ and $27.18 \%$ than $4 \mathrm{R} 1 \mathrm{~B}, 1 \mathrm{R} 1 \mathrm{~B}$ and $2 \mathrm{R} 1 \mathrm{~B}$ treatment respectively. At $45 \mathrm{DAA}$, the content of soluble protein in 8R1B treatment increased by $3.72 \%, 13.13 \%$ and $22.94 \%$ than $1 \mathrm{R} 1 \mathrm{~B}, 2 \mathrm{R} 1 \mathrm{~B}$ and $4 \mathrm{R} 1 \mathrm{~B}$ respectively. At $60 \mathrm{DAA}$, the contents of soluble protein in 1R1B treatment increased by $6.48 \%, 7.18 \%$ and $20.71 \%$ comparing with that treated with $8 \mathrm{R} 1 \mathrm{~B}, 4 \mathrm{R} 1 \mathrm{~B}$ and 2R1B respectively.

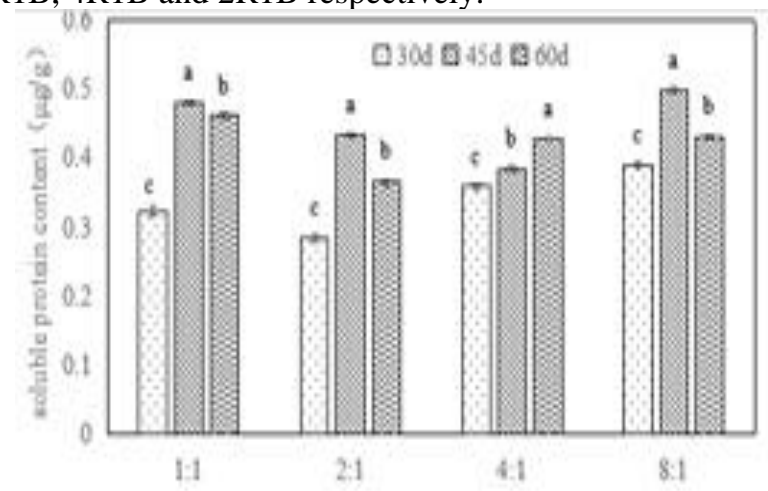

Figure 2. Effects of light quality on soluble protein content in tomato fruit

\section{Effect of Light Quality on The Content of Free Amino Acid in Tomato Fruit}

With tomato fruit development, the contents of free amino acid decreased under 1R1B and 2R1B treatment (Fig. 3 ). During the development of tomato fruit, the content of free amino acid significantly increased at 30,45 and 60 DAA under 1R1B treatment. Comparing with 4R1B, 8R1B and 2R1B treatment, the contents of free amino acid in 1R1B treatment increased by $30.60 \%, 39.52 \%$ and $39.79 \%$ at 30 DAA, and by $18.84 \%, 33.86 \%$ and $47.52 \%$ at 45 DAA, respectively. At 60 DAA, the contents of free amino acid in 1R1B treatment were $18.84 \%, 33.86 \%$ and $47.52 \%$ higher than in $8 \mathrm{R} 1 \mathrm{~B}, 4 \mathrm{R} 1 \mathrm{~B}$ and $2 \mathrm{R} 1 \mathrm{~B}$ treatment, respectively.

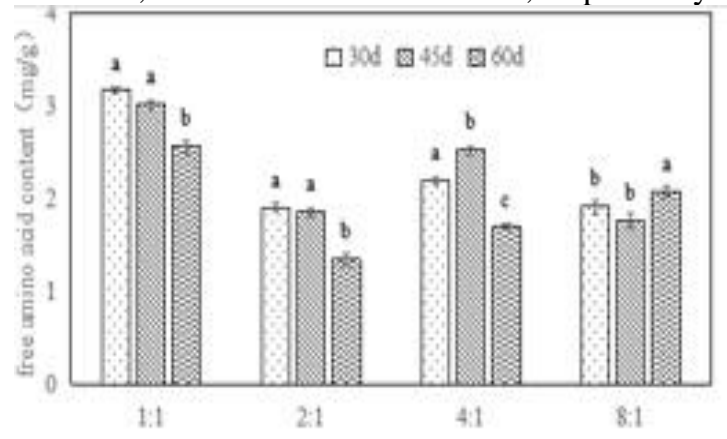

Figure 3. Effects of light quality on free amino acid content in tomato fruit 


\section{Effect of Light Quality on Lycopene Content in Tomato Fruit}

The lycopene contents continuously and significantly increased as tomato fruits developed (Fig. 4). There was no significant difference at 30 DAA among the treatments, while the lycopene content significantly increased at 45 and $60 \mathrm{DAA}$ in 2R1B treatment than other treatment. Comparing with 1R1B, 8R1B and 4R1B treatment, the lycopene contents in $2 \mathrm{R} 1 \mathrm{~B}$ treatment increased by $4.98 \%, 8.19 \%$ and $15.71 \%$ at 45 DAA, and by $16.10 \%, 17.43 \%$ and $24.08 \%$ at 60 DAA, respectively.

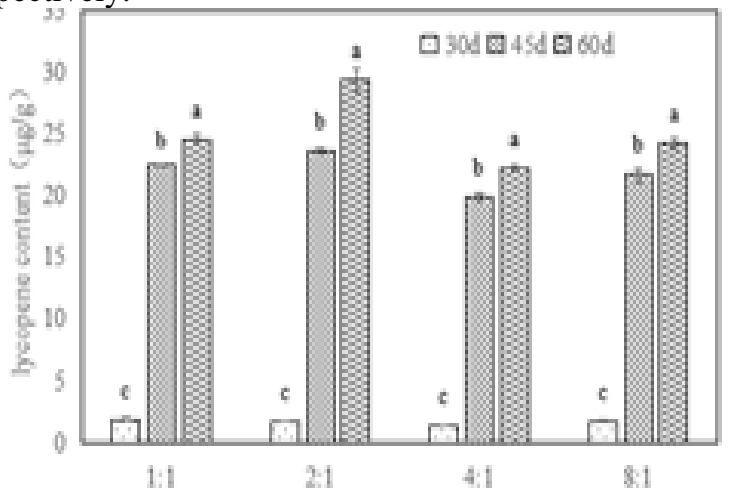

Figure 4. Effects of light quality on lycopene content in tomato fruit

\section{E. Effect of Light Quality on Polyphenol Content in Tomato Fruit}

During the development of tomato fruits, the polyphenol content significantly increased at 45 and 60 DAA under 4R1B and 8R1B treatment (Fig. 5). At 30 DAA, the polyphenol content in 1R1B treatment increased by $8.81 \%$, $9.21 \%$ and $35.15 \%$ than in $4 \mathrm{R} 1 \mathrm{~B}, 8 \mathrm{R} 1 \mathrm{~B}$ and $2 \mathrm{R} 1 \mathrm{~B}$ treatment respectively. At $45 \mathrm{DAA}$, comparing with in $1 \mathrm{R} 1 \mathrm{~B}$ and $2 \mathrm{R} 1 \mathrm{~B}$ treatment, the polyphenol content in 4R1B treatment increased by $24.89 \%$ and $32.63 \%$. However there was the highest polyphenol content in $8 \mathrm{R} 1 \mathrm{~B}$ treatment, which was $6.42 \%, 18.11 \%$ and $26.06 \%$ higher than in $4 \mathrm{R} 1 \mathrm{~B}, 1 \mathrm{R} 1 \mathrm{~B}$ and 2R1B treatment. And at $60 \mathrm{DAA}$, the polyphenol contents in treatments were significantly difference, the contents in 8R1B treatment were $6.42 \%, 18.11 \%$ and $26.06 \%$ higher than in $4 \mathrm{R} 1 \mathrm{~B}, 1 \mathrm{R} 1 \mathrm{~B}$ and $2 \mathrm{R} 1 \mathrm{~B}$ treatment, respectively.

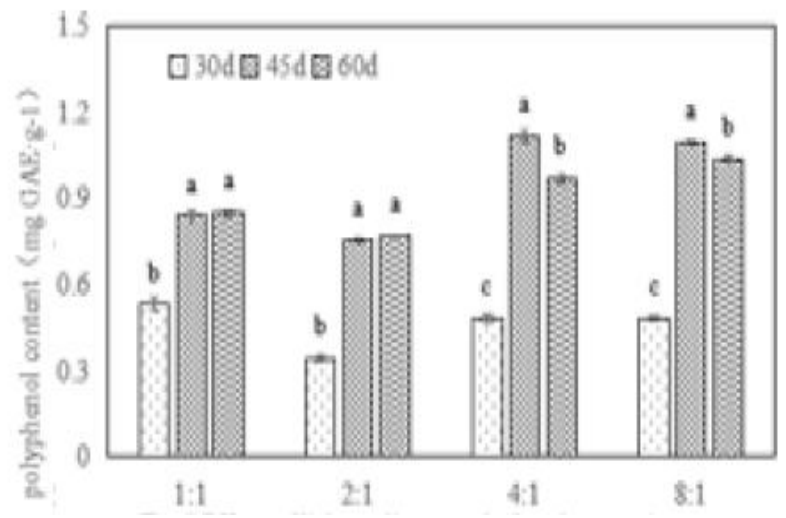

Figure 5. Effects of light quality on polyphenol content in tomato fruit

\section{F. Effect of Light Quality on Flavonoid Content in Tomato Fruit}

With tomato fruit development, the flavonoid contents increased under 1R1B treatment (Fig. 6), while those increased and then decreased under other treatments. At 30 DAA, the flavonoid content in 1R1B treatment increased by $24.29 \%, 30.45 \%$ and $33.04 \%$ than in 2R1B, 4R1B and 8R1B treatment, respectively. At 45 DAA, there was significantly difference in flavonoid contents among treatments, those in $8 \mathrm{R} 1 \mathrm{~B}$ treatment increased by $11.87 \%, 28.42 \%$ and $41.73 \%$ than in 1R1B, 4R1B and 2R1B treatment, respectively. And at $60 \mathrm{DAA}$, the flavonoid contents in treatments were significantly difference, the contents in $1 \mathrm{R} 1 \mathrm{~B}$ treatment were $30.39 \%, 37.78 \%$ and $51.19 \%$ higher than in 4R1B, 8R1B and 2R1B treatment, respectively.

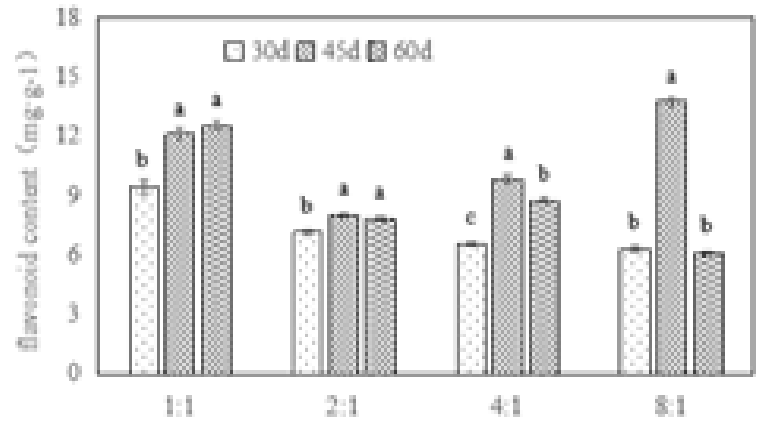

Figure 6. Effects of light quality on flavonoid content in tomato fruit

\section{G. Effect of Light Quality on The Rate of DPPH and FRAP in Tomato Fruit}

During the development of tomato fruits, there was a trend that the rate of DPPH free radical scavenging significantly increased and then were steady (Fig. 7). At 30 DAA, the rate of DPPH in 4R1B treatment increased by $18.98 \%, 32.71 \%$ and $34.50 \%$ than in $1 \mathrm{R} 1 \mathrm{~B}, 8 \mathrm{R} 1 \mathrm{~B}$ and $2 \mathrm{R} 1 \mathrm{~B}$ treatment, respectively. However, there was no significant difference among treatments at 45 DAA, and there was only few difference between 1R1B and 8R1B at 60 DAA.

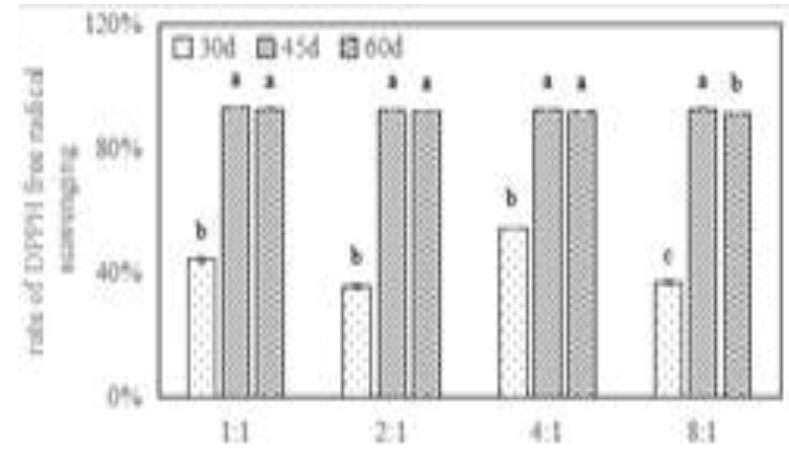

Figure 7. Effects of light quality on rate of DPPH free radical scavenging in tomato fruit

With the tomato fruits developed, FRAP increased and then decreased under treatments (Fig. 8). FRAP significantly increased at 45 and 60 DAA under 1R1B, but significantly increased at 30 DAA under $8 \mathrm{R} 1 \mathrm{~B}$ treatment. At 30 DAA, 
there was no significant difference between $8 \mathrm{R} 1 \mathrm{~B}$ and $4 \mathrm{R} 1 \mathrm{~B}$ treatment, and FRAP in 8R1B treatment increased by $12.27 \%$ and $27.36 \%$ than $1 \mathrm{R} 1 \mathrm{~B}$ and $2 \mathrm{R} 1 \mathrm{~B}$ treatment. At 45 DAA, FRAP in 1R1B treatment was $6.92 \%$ and $17.47 \%$ higher than in $4 \mathrm{R} 1 \mathrm{~B}$ and $2 \mathrm{R} 1 \mathrm{~B}$ treatment. At $60 \mathrm{DAA}$, FRAP in 1R1B treatment increased by $30.39 \%, 37.78 \%$ and $51.19 \%$ than in $2 \mathrm{R} 1 \mathrm{~B}, 8 \mathrm{R} 1 \mathrm{~B}$ and $4 \mathrm{R} 1 \mathrm{~B}$ treatment, respectively.

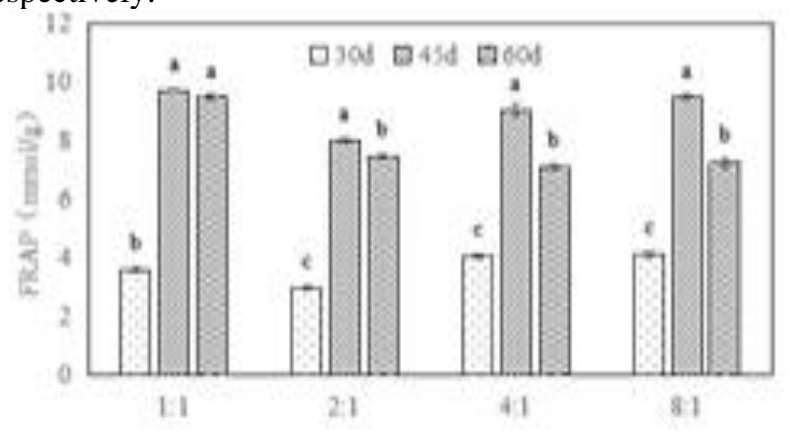

Figure 8. Effects of light quality of FRAP in tomato fruit

\section{DISCUSSION}

Red light promotes the enzyme activity related sugar metabolism by inducing expression of phytochrome gene [17]. The contents of soluble sugar in lettuce increased with the ratio of red LED light increased [3]. The contents of soluble sugar in tomato fruits significantly increased at 30DAA under 4R1B treatment, while increased at 60 DAA under 8R1B treatment, which can be confirmed that red light plays a more important role on the accumulation of soluble sugar.

The contents of free amino acids and soluble protein increased under Blue light treatment in alfalfa sprouts [6] and Chinese toon sprouts [7].The contents of free amino acid and soluble protein significantly increased during the development of tomato fruits under 1R1B treatment. Under blue light, dark respiration significantly enhanced, could provide carbon frame for the synthesis of organic nitrogen compounds, and then the synthesis of free amino acid and soluble protein increased [18]. And the activities of nitrate reductase could be enhanced by blue light, thus provided more nitrogen for protein synthesis $[19,20]$.

Lycopene is the important pigment in tomato fruits. The lycopene contents significantly increased in tomato fruit under red light [10], however significantly increased in cherry tomato fruit under blue light [21]. With tomato fruits development, the lycopene contents continuously and significantly increased, and significantly increased from 45 to 60 DAA under 2R1B treatment. Phytoene synthase (PSY) is the main rate-determining enzyme in the carotenoid biosynthesis pathway, and up-regulation of PSY was mediated by light through phytochrome, PSY was induced significantly in red light conditions [22-24].

With higher blue light ratio all phenolic acids and flavonoids increased in roses, chrysanthemums, and campanulas [25].The polyphenol content significantly increased at 45 and $60 \mathrm{DAA}$ under $4 \mathrm{R} 1 \mathrm{~B}$ or $8 \mathrm{R} 1 \mathrm{~B}$ treatment, and significantly increased at 30,45 and 60 DAA under $1 \mathrm{R} 1 \mathrm{~B}$, but significantly increased at 45 DAA under 8R1B treatment.

Shorter wavelengths, in the range of blue and UV-light show the most prominent effect in the accumulation of flavonoids in fruits, often by increasing the expression of flavonoid pathway genes. Flavonoid contents in tomato fruit significantly increased at 30, 45 and 60 DAA under 1R1B, but significantly increased at 45 DAA under 8R1B treatment. Flavonoid content in alfalfa sprouts significantly increased under blue light treatment [9]. In unripe strawberries, blue light increased significantly the biosynthesis of anthocyanins and the expression of FaCHS [26] Fruit-specific suppression of an endogenous photomorphogenesis regulatory gene (DET1) by light resulted in enhanced carotenoid accumulation and increased flavonoid levels in tomato fruits [27]. Carotenogenic genes and carotenoid accumulation were both induced by blue light treatment in citrus juice sacs in vitro [28] and by red light in citrus fruits [29]. However, variation in response can be high between and even within species.

The rate of DPPH only significantly increased in 4R1B treatment at 30 DAA, while FRAP significantly increased at 45 and 60 DAA under 1R1B, but significantly increased at 30 DAA under 8R1B treatment. The increase in contents of total phenol and flavonoid by the LED treatments has a strong positive correlation with the enhancement of antioxidant capacity [30]. Red and blue compound light affects antioxidant capacity and radical scavenging potential by affecting the contents of polyphenol and flavonoid.

Thus, during tomato fruit development, the nutritional quality was significantly affected by supplemental Red and Blue light. The contents of soluble protein, free amino acid, flavonoid and ferric reducing antioxidant potential (FRAP) significantly increased under the 1R1B (Red : Blue $=1: 1$ ) treatment, and the contents of soluble sugar and polyphenol, the rate of DPPH free radical scavenging significantly increased under the 4R1B (Red: Blue $=4: 1)$ treatment, the lycopene contents significantly increased under 2R1B (Red : Blue $=2: 1$ ) treatment.

\section{ACKNOWLEDGMENTS}

This work was supported by Teamwork Projects Funded by Guangdong Natural Science Foundation (No. S2013030012842), Guangdong Provincial Science \& Technology Project (No. 2015B090903074), and the Guangzhou Science \& Technology Project (201605030005).

\section{REFERENCE}

[1] R. M. Wheeler, C. L. Mackowiak, J. C. Sager, "Soybean stem growth under high-pressure sodium with supplemental blue lighting," Agronomy Journal, 1991, 83(5): 903-906.

[2] D. T. Nhut, T. Takamura, H. Watanabe, K. Okamoto, M. Tanaka, "Responses of strawberry plantlets cultured in vitro under superbright red and blue light-emitting diodes (LEDs)," Plant Cell Tissue \& Organ Culture, 2003, 73(1): 43-52.

[3] R. Chen, H. Liu, S. Song, G. Sun, R. Chen, "Effects of light quality on growth and quality of lettuces in hydroponic," 12th China International Forum on Solid State Lighting, SSLCHINA 2015 p 154-156 
DOI:10.1109/ SSLCHINA. 2015. 7360712

[4] Y. Yu, Q. Yang, J. Zhao, W. Liu, "Spectral absorban, cegrowth and nutritional quality responses of three leaf-color lettuce cultivars to LED light qualities," China Illuminating Engineering Journal, 2013(S1): 139-145.(In Chinese)

[5] X. Liu, X. Jiao, Z. Xu, Y. Yang, "Effects of red and blue LED on growth, nutritional quality and nitrate nitrogen content of lettuce," Journal of Nanjing Agricultural University,2013(5): 139-143.(In Chinese)

[6] L. Tang, Y. Lu, J. Cui, "Effects of light quality on nutritional quality and antioxidant properties in alfalfa sprouts," Food Science, 2014, 35(13): 32-36. (In Chinese)

[7] Y. Lu, L. Tang, X. Zhang, "Effects of light quality on growth and nutritional quality in Chinese toon sprouts," Journal of Nutrition, 2014, 36(2): 188-192. (In Chinese)

[8] G. Samuolienè, R. Sirtautas, A. Brazaityte, P. Duchovskis, "LED lighting and seasonality effects antioxidant properties of baby leaf lettuce," Food Chemistry, 2012, 134(3): 1494-1499.

[9] K. Son, M. Oh, "Leaf shape, growth, and antioxidant phenolic compounds of two lettuce cultivars grown under various combinations of blue and red light-emitting diodes," Hortscience. 2013, 48(8): 988-995.

[10] Q. Chen, S. Liu, Z. Zhang, H. Cui, S. Hao, Z. Liu, "Effect of different light emitting diode sources on tomato fruit quality during color-changed period," Transactions of the CSAE, 2009,25(5): 156-161 (In Chinese)

[11] K. Canene-Adams, J. K. Campbell, S. Zaripheh, E. H. Jeffery, J. W., JR. Erdman, "The tomato as a functional food," Journal of Nutrition, 135, 2005: 1226-1230.

[12] H. Li, "Principle and technology of physiological and biochemical experiment," Higher education press, 2000, pp.184-185, 192-194 (in Chinese).

[13] L. Helyes, A. Lugasi, Z. Pék, "Effect of natural light on surface temperature and lycopene content of vine ripened tomato fruit," Can. J. Plant Sci., 2007, 87: 927-929.

[14] B. Tadolini, C. Juliano, L. Piu, F. Franconi, L. Cabrini, "Resveratrol inhibition of lipid peroxidation," Free Radical Research, 2000, 33(1): $105-114$

[15] G. Ling, "Antioxidant food and Health," Chemical industry press, 2004, pp 121-123 (In Chinese)

[16] I. F. F. Benzie, J. J. Strain, "Ferric reducing/antioxidant power assay: Direct measure of total antioxidant activity of biological fluids and modified version for simultaneous measurement of total antioxidant power and ascorbic acid concentration," Methods in Enzymology. 1999, 299(1): 15-27.

[17] M. J. Kasperbauer, "Strawberry yield over red versus black plastic mulch," Crop Science, 2000, 40(1): 171-174.
[18] W. Kowallik, "Blue light effects on respiration," Annual Review of Plant Physiology. 1982, 33(1): 51-72.

[19] H. Ninnemann, "Some aspects of blue light research during the last decade," Photochemistry and photobiology, 1995, 61(1): 22-31.

[20] W. H. Campbell, "Nitrate reductase biochemistry comes of age," Plant Physiology, 1996, 111(2): 355-361.

[21] X. Liu, T. Chang, S. Guo, Z. Xu, W. Chen, "Effect of irradiation with blue and red LED on fruit quality of cherry tomato during growth period," Chinese Vegetables. 2010, 2010(22): 21-27. (In Chinese)

[22] J. Hirschberg, "Carotenoid biosynthesis in flowering plants," Current opinion in plant biology, 2001, 4(3): 210-218.

[23] L. O. Lindgren, K. G. Stålberg, A. Höglund, "Seed-specific overexpression of an endogenous Arabidopsis phytoene synthase gene results in delayed germination and increased levels of carotenoids, chlorophyll, and abscisic acid," Plant physiology, 2003, 132(2): 779-785.

[24] W. Lee, J. Huang, L. Chen, C. Tsai, F. Chen, "Developmental and LED light source modulation of carotenogenic gene expression in Oncidium gower ramsey flowers," Plant molecular biology reporter, 2013, 31(6): 1433-1445.

[25] T. Ouzounis, X. Fretté, E. Rosenqvist, C. Ottosenc, "Spectral effects of supplementary lighting on the secondary metabolites in roses, chrysanthemums, and campanulas," Journal of plant physiology, 2014, 171(16): 1491-1499.

[26] Y. Kadomura-Ishikawa, K. Miyawaki, S. Noji, A. Takahashi, "Phototropin2 is involved in blue light-induced anthocyanin accumulation in Fragaria $\times$ ananassa fruits," J. Plant Res. 2013, 126, 847-857.

[27] G.R. Davuluri, A. van Tuinen, P.D. Fraser, A. Manfredonia, R Newman, D. Burgess, D. A. Brummell, S. R. King, J. Palys, J. Uhlig, P. M. Bramley, H. M. Pennings, C. Bowler, " Fruit-specific RNAimediated suppression of DET1 enhances carotenoid and flavonoid content in tomatoes," Nat Biotechnol, 2005, 23:890-895

[28] L. Zhang, G. Ma, M. Kato, K. Yamawaki, T. Takagi, Y. Kiriiwa, Y. Ikoma, H. Matsumoto, T. Yoshioka, H. Nesumi, "Regulation of carotenoid accumulation and the expression of carotenoid metabolic genes in citrus juice sacs in vitro," J. Exp. Bot., 2012, 63:871-886

[29] G. Ma, L. Zhang, M. Kato, K. Yamawaki, Y. Kiriiwa, M. Yahata, Y. Ikoma, H. Matsumoto, "Effect of blue and red LED light irradiation on beta-cryptoxanthin accumulation in the flavedo of citrus fruits," $\mathrm{J}$. Agric. Food Chem., 2012,60:197-201

[30] A. Manivannan, P. Soundararajan, N. Halimah, H. K. Chung, R. J. Byoung, "Blue LED light enhances growth, phytochemical contents, and antioxidant enzyme activities of Rehmannia glutinosa cultured in vitro," Horticulture, Environment, and Biotechnology, 2015, 56(1): 105-113. 